\title{
Liver Fibrosis-Novel Therapy Perspective
}

\author{
Panakanti R* \\ Roosevelt University College of Pharmacy, USA \\ *Corresponding author: Ravikiran Panakanti, Roosevelt University College of \\ Pharmacy, 1400 N. Roosevelt Blvd, SCH 250, Schaumburg, IL 60173, USA, E-mail: \\ rpanakanti@roosevelt.edu
}

\section{Mini Review \\ Volume 1 Issue 7}

Received Date: July 31, 2017

Published Date: November 02, 2017

\begin{abstract}
Liver fibrosis is highly prevalent in United States. No therapy has been successful to cure it so far. The current approaches in treatment are to prevent its causative etiology such as viral infection, alcohol abuse etc. or liver transplantation. Much progress has been made to understand the mechanisms for development of myofibroblasts in developing fibro genesis, inflammation and cell death. This review summarizes some of the key aspects of liver fibrosis and some novel therapies that have a potential to become a cure.
\end{abstract}

Keywords: Chemokines; Liver fibrosis; Hepatoprotection; Hepatocytes

Abbreviations: NASH: Nonalcoholic Steatohepatitis; ECM: Extracellular Matrix; IASL: International Association for the Study of the Liver; NAFLD: Nonalcoholic Fatty Liver Disease; HSCs: Hepatic Stellate Cells; LSECs: Liver Sinusoidal Endothelial Cells; TFO: Triplex Forming Oligonucleotide; CBDL: Common Bile Duct Ligation; BECs: Biliary Epithelial Cells

\section{Introduction}

Liver fibrosis is a consequence of chronic liver injury, viral infection, alcohol abuse, toxins, nonalcoholic steatohepatitis (NASH), cancer growth, immune related processes and other factors [1]. It basically results in the excessive accumulation of extracellular matrix (ECM) in the liver thereby causing chronic damage to the liver [2]. It is estimated that around 3.9 million people in United States are suffering from chronic liver disease. There are around 12 deaths for every 100,000 population [3]. There is no cure for liver fibrosis yet. The only option for patients with advanced stage of liver fibrosis is liver transplantation. However, the biggest challenge with liver fibrosis is that it remains mostly unnoticed especially in patients with Nonalcoholic fatty liver disease (NAFLD). Liver biopsy is typically done to assess the condition of the liver. Scientists have come up with different systems of classification to score or grade the level of inflammation or fibrosis and the stage of liver fibrosis [411]. Out of these the French METAVIR, the Batts-Ludwig, the International Association for the Study of the Liver (IASL) and the Ishak Scoring systems are commonly used to assess the grade of inflammation and the stage of liver fibrosis [11]. Off late non-invasive diagnostic tests coupled with various imaging techniques are considered to be beneficial in diagnosing liver fibrosis/ cirrhosis and can reduce the need for performing biopsies [12]. Also, these methods can help in early diagnosis of fibrosis which is beneficial for treating it [13].

Liver fibrosis is considered as the healing process of the liver following of its injury. This however leads to formation of permanent scarring to the liver which can further advance to cirrhosis, liver failure and portal hypertension [14]. Scarring prevents effective liver regeneration and hinders normal liver function [1]. The term fibrosis basically defines the effect of inflammation on liver. Inflammation and cell death are the key parameters playing a major role in the onset of liver fibrosis [15]. But it is important to note that it's the liver fibrosis that generally progresses to cirrhosis and is the 


\section{Open Access Journal of Pharmaceutical Research}

major cause of morbidity. Hence, it is very important to understand the etiology and pathogenesis of fibrosis to develop a proper therapy or prevent its progression to irreversible cirrhosis. Liver fibrosis results in the excessive accumulation of extracellular matrix (ECM). This is mainly due to the hepatic stellate cells (HSCs) getting activated, resulting in inflammation of hepatocytes causing excessive accumulation of Type I collagen and ECM. These activated HSCs are major source of myofibroblasts and these are effector cells of fibro genesis, followed by portal fibroblasts $[16,17]$. Myofibroblasts further cause increased synthesis of type I and III fibrillar collagen resulting in accumulation of ECM [18]. Myofibroblasts lead to accumulation of ECM by secreting cytokines like transforming growth factor-beta (TGF- $\beta 1$ ), tumor necrosis factor-alpha (TNF- $\alpha$ ), interleukin-1 beta (IL-1 $\beta$ ), interferon gamma (IFN- $\gamma$ )etc. along with chemokines such as CCL-2, CCL-5, CXCL-10 and other growth factors. Hepatocyte death due to liver injury, viral infection or other causes is considered to be the main inducer of inflammation and liver fibrosis. Any injury to the liver due any of the factors mentioned above can lead to macrophages, NK cells, platelet derived factors that activate HSCs and subsequently cause inflammation, cell death and fibrosis. Liver sinusoidal endothelial cells (LSECs) can also activate HSCs in fibrotic liver [15]. This has been evident in bile duct ligated animal fibrotic models.

\section{Liver Fibrosis Therapy}

There are several potential therapies aimed at several targets including liver injury, inflammation, prevention of activation of HSCs, prevention of apoptosis of hepatocytes, inhibiting proliferation of HSCs, stimulating the degradation of ECM, interruption of ECM accumulation, inhibiting type I collagen etc. Other approaches such as treating the viral infection, prevention of alcohol abuse etc. can also lead to cure/reversal of fibrosis and lead to proper regeneration of liver.

We have used a triplex forming oligonucleotide (TFO) that will inhibit the type $\alpha 1$ (I) collagen. Common Bile duct ligation (CBDL) is the most frequently used method in developing fibrosis. Biliary fibrosis can be occurred either due to biliary atresia or primary sclerosing cholangitis. The injury to the bile duct by performing BDL may trigger a chain of events such as release of inflammatory cytokines, chemokines and LSECs etc., resulting in the development of fibrosis. Further the biliary epithelial cells would get transformed into mesenchymal cells (EMT) resulting in the proliferation of myofibroblasts. We have evaluated the use of TFOs inhibiting Type $\alpha 1$ (I) collagen in attenuating fibrosis induced by bile duct ligation. CBDL results in the transformation of biliary epithelial cells (BECs) to $\alpha$ smooth muscle act in ( $\alpha$-SMA) expressing myofibroblasts. Treatment with TFO showed a significant reduction in the TGF- $\beta 1$ levels and other pro-inflammatory cytokines. There was also a decrease in the $\alpha$-SMA levels in the TFO treated group compared to the non-treated CBDL group suggesting that the inhibition of collagen can also lead to down regulation of $\alpha$-SMA levels. TFO treatment successfully inhibited the transcription of Type I collagen, showed depreciation of fibrosis, attenuation of EMT in bile duct ligated animal models [18]. We have also used hedgehog inhibitors (cyclopamine and GDC-0449) that were successful in attenuating liver fibrosis in BDL rats $[19,20]$. Mahato, et al. have used a hedgehog inhibitor GDC-0449 and micro RNA (miR-29b1) simultaneously to treat liver fibrosis. Mir-29b1 selectively targets profibriotic genes and their use showed decreased collagen levels, enhanced hepatoprotection in CBDL induced liver fibrosis in mice [21].

TGF- $\beta 1$ silencing using a siRNA showed promise in mitigating liver fibrosis. Specific targeting of TGF- $\beta 1$ mRNA with small interfering RNA (siRNA) and short hairpin RNA (shRNA) in hepatic stellate cells (HSC-T6), showed a significant inhibition of TGF- $\beta 1$, tissue inhibitor of metalloproteinase 1 (TIMP-1), $\alpha$-smooth muscle act in $(\alpha-S M A)$ and type I collagen expression [22]. This can be an alternative strategy to inhibit various inflammatory cytokines that have been elucidated to show a major role in the development of liver fibrosis. To corroborate the significance of TGF- $\beta 1$ in activation of hepatic stellate cells, it has been recently shown that gremlin-1, one of the target genes of TGF- $\beta 1$ signaling pathway has shown to enhance the activation of HSCs through over expression of TGF- $\beta$ [23]. NOD-like receptor family CARD domain containing 5, (NLRC5) has shown to up regulate liver fibrosis by TGF- $\beta 1 /$ Smad and nuclear factor-kappa B (NF$\kappa B)$ signaling pathways [24]. Benz et al have shown that micro RNAs miR-30 and miR-193 play a role in activating the HSCs by regulating TGF- $\beta$ expression [25]. However, these are relatively new therapies and much more studies have to done to ascertain their beneficial effects.

\section{Conclusion}

Liver fibrosis is a difficult disease to cure. It is still unknown when liver fibrosis would become irreversible. There are so many factors at play because of which targeting one aspect of the disease is not sufficient. It is important to have a connection between the pathways of inflammation, cell death and liver fibrosis. Much progress 


\section{Open Access Journal of Pharmaceutical Research}

has been made in developing antifibrotic therapies [26] and hopefully it will lead to a cure in nearby future. By using a combinatorial therapy of hedgehog inhibitors and gene therapy to specifically target and inhibit inflammatory genes can probably help in mitigating liver fibrosis.

\section{References}

1. Bataller R, DA Brenner (2005) Liver fibrosis. J Clin Invest 115(2): 209-218.

2. Friedman SL (2003) Liver fibrosis -- from bench to bedside. J Hepatol 38 (S1): 38-53.

3. https://www.cdc.gov/nchs/fastats/liver-disease.htm

4. Goodman ZD (2007) Grading and staging systems for inflammation and fibrosis in chronic liver diseases. J Hepatol 47(4): 598-607.

5. Scheuer PJ (1991) Classification of chronic viral hepatitis: a need for reassessment. J Hepatol 13(3): 372-374.

6. Knodell RG, Ishak KG, Black WC, Chen TS, Craig R, et al. (1981) Formulation and application of a numerical scoring system for assessing histological activity in asymptomatic chronic active hepatitis. Hepatology 1(5): 431-435.

7. Desmet VJ, Gerber M, Hoofnagle JH, Manns M, Scheuer PJ (1994) Classification of chronic hepatitis: diagnosis, grading and staging. Hepatology 19(6): 1513-1520.

8. Bedossa P, Poynard T (1996) An algorithm for the grading of activity in chronic hepatitis C. The METAVIR Cooperative Study Group. Hepatology 24(2): 289-293.

9. Batts KP, Ludwig J (1995) Chronic hepatitis. An update on terminology and reporting. Am J Surg Pathol 19(12): 1409-1417.

10. Ishak K, Baptista A, Bianchi L, Callea F, De Groote J, et al. (1995) Histological grading and staging of chronic hepatitis. J Hepatol 22(6): 696-699.

11. Ghany MG, Strader DB, Thomas DL, Seeff LB (2009) Diagnosis, management, and treatment of hepatitis C: an update. Hepatology 49(4): 1335-1374.

12. Lurie $Y$, Webb M, Kuint RC, Shteingart S, Lederkremer GZ (2015) Non-invasive diagnosis of liver fibrosis and cirrhosis. World J Gastroenterol 21(41): 1156711583.

13. Stal P (2015) Liver fibrosis in non-alcoholic fatty liver disease - diagnostic challenge with prognostic significance. World J Gastroenterol 21(39): 1107711087.

14. Kisseleva T, Brenner DA (2011) Anti-fibrogenic strategies and the regression of fibrosis. Best Pract Res Clin Gastroenterol 25(2): 305-317.

15. Seki E, Schwabe RF (2015) Hepatic inflammation and fibrosis: functional links and key pathways. Hepatology 61(3): 1066-1079.

16. Koyama Y, Xu J, Liu X, Brenner DA (2016) New Developments on the Treatment of Liver Fibrosis. Dig Dis 34(5): 589-596.

17. Elpek GO (2014) Cellular and molecular mechanisms in the pathogenesis of liver fibrosis: An update. World J Gastroenterol 20(23): 7260-7276.

18. PanakantiR, Pratap A, Yang N, Jackson JS, Mahato RI (2010) Triplex forming oligonucleotides against type alpha1 (I) collagen attenuates liver fibrosis induced by bile duct ligation. Biochem Pharmacol 80(11): 1718-1726.

19. Pratap A, Singh S, Mundra V, Yang N, Panakanti R, et al. (2012) Attenuation of early liver fibrosis by pharmacological inhibition of smoothened receptor signaling. J Drug Target 20(9): 770-782.

20. Pratap A, Panakanti R, Yang N, Lakshmi R, Modanlou KA, et al. (2011) Cyclopamine attenuates acute warm ischemia reperfusion injury in cholestatic rat liver: hope for marginal livers. Mol Pharm 8(3): 958-968.

21. Mahato RI, Kumar V, Mondal G, Dutta R, (2016) Codelivery of small molecule hedgehog inhibitor and miRNA for treating liver fibrosis. Biomaterials 76 : 144-156.

22. Cheng K, N Yang, Mahato RI (2009) TGF-beta1 gene silencing for treating liver fibrosis. Mol Pharm 6(3): 772-779.

23. Zhang YQ, Wan LY, He XM, Ni YR, Wang C, et al. (2017) Gremlin1 Accelerates Hepatic Stellate Cell Activation through Upregulation of TGF-Beta Expression. DNA Cell Biol 36(7): 603-610. 


\section{Open Access Journal of Pharmaceutical Research}

24. Xu T, Ni MM, Xing-Li, Li XF, Meng XM, et al. (2016) NLRC5 regulates TGF-beta1-induced proliferation and activation of hepatic stellate cells during hepatic fibrosis. Int J Biochem Cell Biol 70: 92-104.

25. Roy S, Benz F, Vargas Cardenas D, Vucur M, Gautheron J, et al. (2015) miR-30c and miR-193 are a part of the TGF-beta-dependent regulatory network controlling extracellular matrix genes in liver fibrosis. J Dig Dis 16(9): 513-524.

26. Zhang CY, Yuan WG, He P, Lei JH, Wang CX (2016) Liver fibrosis and hepatic stellate cells: Etiology, pathological hallmarks and therapeutic targets. World J Gastroenterol 22(48): 10512-10522. 